\title{
$\rho$ mesons in strong abelian magnetic field in SU(3) lattice gauge theory
}

\section{E.V. Luschevskaya}

Institute of Theoretical and Experimental Physics, Bolshaya Cheremushkinskaya 25, Moscow, 117218, Russia

E-mail: luschevskaya@itep.ru

\section{O.A. Kochetkov}

Institute of Theoretical and Experimental Physics, Bolshaya Cheremushkinskaya 25, Moscow, 117218, Russia

Institut fur Theoretische Physik, Universitat Regensburg, D-93040 Regensburg, Germany

E-mail: oleg.kochetkov@physik.uni-r.de

\section{O.V. Larina*}

Institute of Theoretical and Experimental Physics, Bolshaya Cheremushkinskaya 25, Moscow, 117218, Russia

E-mail: olarina@itep.ru

\section{O.V. Teryaev}

Joint Institute for Nuclear Research, Dubna, Russia

E-mail: teryaevetheor.jinr.ru

\begin{abstract}
We explore the masses of neutral and charged $\rho$ mesons in strong abelian magnetic field in $S U(3)$ gluodynamics. The behaviour of the ground state energy of these particles in the external magnetic field depends on its spin projection $s_{z}$ on the axis of external magnetic field. The masses of $\rho^{0}$ meson with $s_{z}= \pm 1$ increase with the field. The masses of $\rho^{ \pm}$mesons with zero spin also grow with the magnetic field. The ground state energies of $\rho^{-}$meson with $s_{z}=-1$ and $\rho^{+}$meson with $s_{z}=+1$ diminish as a function of the field, while the energies of $\rho^{+}$meson with $s_{z}=-1$ and $\rho^{-}$meson with $s_{z}=+1$ rise with the field value.
\end{abstract}

The 32nd International Symposium on Lattice Field Theory,

23-28 June, 2014

Columbia University New York, NY

\footnotetext{
* Speaker.
} 


\section{Introduction}

The exploration of hadronic matter in strong abelian magnetic field has a fundamental meaning. The strong magnetic fields of $\sim 2 \mathrm{GeV}$ may be associated with the formation of the Early Universe [1]. Now it is possible to observe such magnetic fields in non-central heavy-ion collisions in terrestrial laboratories (ALICA, RHIC, NICA, FAIR). The field value can reach up to $15 m_{\pi}^{2} \sim 0.27 \mathrm{GeV}^{2}$ [2], i.e. the order of energies at which the QCD effects appear.

Quantum chromodynamics in a strong magnetic field shows a lot of bright interesting effects, e.g. inverse magnetic catalysis [3]. Calculations on the lattices are a good method for study of the phase diagram of QCD in external magnetic field $[4,5,6]$. Numerical simulations in QCD with $N_{f}=2$ and $N_{f}=2+1$ show that strongly interacting matter in strong magnetic field posses paramagnetic properties in the confinement and deconfinement phases [7, 8, 9]. Equation of state for quark-gluon plasma in strong magnetic field was also investigated in [10].

In the framework of the Nambu-Jona-Lasinio model it was shown that QCD vacuum becomes a superconductor in sufficiently strong magnetic field $\left(B_{c}=m_{\rho}^{2} / e \simeq 10^{16} \mathrm{Tl}\right)[11,12]$ along the direction of the magnetic field. This transition to superconducting phase is accompanied by a condensation of charged $\rho$ mesons, their masses turns to zero.

We have calculated the ground state energies of neutral and charged vector mesons as a function of external magnetic field depending on the spin projections $s_{z}=0,+1$ or -1 on the axis of the magnetic field in $S U$ (3) lattice gauge theory without dynamical quarks. For not very large magnetic fields our data for charged particles confirm the picture of Landau levels for pointlike particles. Articles [13] [14] and [15] are also devoted to the behaviour of meson masses in the external abelian magnetic field.

\section{Observables and details of calculations}

The technical details of our calculations are presented in [16]. We generate $S U(3)$ statistically independent lattice gauge configurations with tadpole improved Symanzik action [17]. Then we solve Dirac equation numerically

$$
D \psi_{k}=i \lambda_{k} \psi_{k}, \quad D=\gamma^{\mu}\left(\partial_{\mu}-i A_{\mu}\right)
$$

and find eigenfunctions $\psi_{k}$ and eigenvectors $\lambda_{k}$ for a test quark in the external gauge field $A_{\mu}$, which is a sum of non-abelian $S U(3)$ gluonic field and $U(1)$ abelian constant magnetic field.

We add the abelian magnetic field only into Dirac operator, because our theory doesn't contain dynamical quarks. Our simulations has been carried out on symmetrical lattices with lattice volumes $16^{6}, 18^{4}$ and lattice spacings $a=0.105 \mathrm{fm}, a=0.115 \mathrm{fm}, 0.125 \mathrm{fm}$. The number of configurations for the lattices with $a=0.115 \mathrm{fm}$ and $0.125 \mathrm{fm}$ spacings were $200-300$, for the finest lattice with $a=0.105 \mathrm{fm} 95$ configurations was used.

We calculate the correlation functions in coordinate space

$$
\left\langle\psi^{\dagger}(x) O_{1} \psi(x) \psi^{\dagger}(y) O_{2} \psi(y)\right\rangle_{A},
$$

where $O_{1}, O_{2}=\gamma_{\mu}, \gamma_{v}$ are Dirac gamma matrices, $\mu, v=1, . ., 4$ are Lorenz indices. 
In order to calculate the observables (2.2) the quark propagators have to be computed. The Dirac propagator for the massive quark is

$$
D^{-1}(x, y)=\sum_{k<M} \frac{\psi_{k}(x) \psi_{k}^{\dagger}(y)}{i \lambda_{k}+m} .
$$

where $M=50$ is the number of the lowest eigenmodes. For the correlators (2.2) the following equality is fulfilled

$$
\left\langle\bar{\psi} O_{1} \psi \bar{\psi} O_{2} \psi\right\rangle_{A}=-\operatorname{tr}\left[O_{1} D^{-1}(x, y) O_{2} D^{-1}(y, x)\right]+\operatorname{tr}\left[O_{1} D^{-1}(x, x)\right] \operatorname{tr}\left[O_{2} D^{-1}(y, y)\right] .
$$

We calculate the correlators and the make its Fourier transformation. For the meson ground state we have to choose $\langle\mathbf{p}\rangle=0$. For particles with zero momentum their energy is equal to its mass $E_{0}=m_{0}$. The expansion of correlation function to exponential series has the form

$$
\tilde{C}\left(n_{t}\right)=\left\langle\psi^{\dagger}\left(\mathbf{0}, n_{t}\right) O_{1} \psi\left(\mathbf{0}, n_{t}\right) \psi^{\dagger}(\mathbf{0}, 0) O_{2} \psi(\mathbf{0}, 0)\right\rangle_{A}=\sum_{k}\left\langle 0\left|O_{1}\right| k\right\rangle\left\langle k\left|O_{2}^{\dagger}\right| 0\right\rangle e^{-n_{t} a E_{k}},
$$

where $a$ is the lattice spacing, $n_{t}$ is the number of nodes in the time direction, $E_{k}$ is the energy of the state with quantum number $k$. From expansion (2.5) one can see that for large $n_{t}$ the main contribution comes from the ground state. Because of periodic boundary conditions on the lattice the main contribution to the ground state has the following form

$$
\tilde{C}_{f i t}\left(n_{t}\right)=A_{0} e^{-n_{t} a E_{0}}+A_{0} e^{-\left(N_{T}-n_{t}\right) a E_{0}}=2 A_{0} e^{-N_{T} a E_{0} / 2} \cosh \left(\left(N_{T}-n_{t}\right) a E_{0}\right),
$$

where $A_{0}$ is a constant, $E_{0}$ is the energy of the ground state.

Mass of the ground state can be evaluated fitting the correlator (2.5) with (2.6) function. In order to minimize the errors and exclude the contribution of excited states we take various values of $n_{t}$ from the interval $5 \leq n_{t} \leq N_{T}-5$.

\section{Results}

The correlators of vector currents in various spatial dimensions have the following form

$$
\begin{aligned}
& C_{x x}^{V V}=\left\langle\bar{\psi}\left(\mathbf{0}, n_{t}\right) \gamma_{1} \psi\left(\mathbf{0}, n_{t}\right) \bar{\psi}(\mathbf{0}, 0) \gamma_{1} \psi(\mathbf{0}, 0)\right\rangle, \\
& C_{y y}^{V V}=\left\langle\bar{\psi}\left(\mathbf{0}, n_{t}\right) \gamma_{2} \psi\left(\mathbf{0}, n_{t}\right) \bar{\psi}(\mathbf{0}, 0) \gamma_{2} \psi(\mathbf{0}, 0)\right\rangle, \\
& C_{z z}^{V V}=\left\langle\bar{\psi}\left(\mathbf{0}, n_{t}\right) \gamma_{3} \psi\left(\mathbf{0}, n_{t}\right) \bar{\psi}(\mathbf{0}, 0) \gamma_{3} \psi(\mathbf{0}, 0)\right\rangle .
\end{aligned}
$$

The energy of the ground state or the mass of vector $\rho$ meson with $s_{z}=0$ spin projection could be obtained from the $C_{z z}^{V V}$ correlator. The combinations of correlators

$$
C^{V V}\left(s_{z}= \pm 1\right)=C_{x x}^{V V}+C_{y y}^{V V} \pm i\left(C_{x y}^{V V}-C_{y x}^{V V}\right) .
$$

correspond to the vector particles with quantum numbers $s_{z}=+1$ and $s_{z}=-1$.

In Fig. 1 we see the mass of the state with zero spin which was obtained from the correlator $C_{z z}^{V V}$. We expect that at small magnetic field this state corresponds to the neutral $\rho^{0}$ meson with zero spin projection on the axis $z$. The mass of the state diminishes with the magnetic field at small 


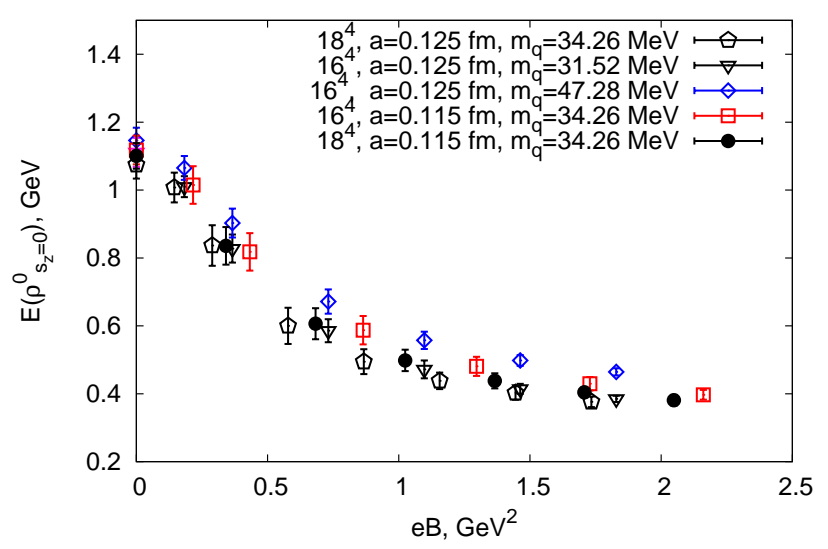

Figure 1: The ground state energy of the neutral $\rho^{0}$ meson with spin $s_{z}=0$ as a function of magnetic field for lattice volume $16^{4}$ and $18^{4}$, lattice spacings $a=0.115 \mathrm{fm}$ and $0.125 \mathrm{fm}$ and various bare quark masses.

$e B$. In nature at strong magnetic field the branching for the decay $\rho^{0} \rightarrow \pi^{0} \gamma$ have to be large. It is not easy to differ between $\rho^{0}\left(s_{z}=0\right)$ and $\pi^{0}(s=0)$ on the lattice because they have the same quantum numbers. We do not make it here.

Fig. 2 shows the mass of the neutral $\rho^{0}$ meson with spin projections $s_{z}= \pm 1$. The masses for $s_{z}=-1$ and $s_{z}=+1$ grow with the field value and coincide with each other because the imaginary part of (3.4) is zero. This is a manifestation of C-parity of $\rho^{0}$ meson. From Fig.1 and 2 we see that the data cohere for $16^{4}$ and $18^{4}$ lattice volumes and the same lattice spacings $a=0.115 \mathrm{fm}$. Lattice spacing effects are also not large. The results are in qualitative agreement with the calculations

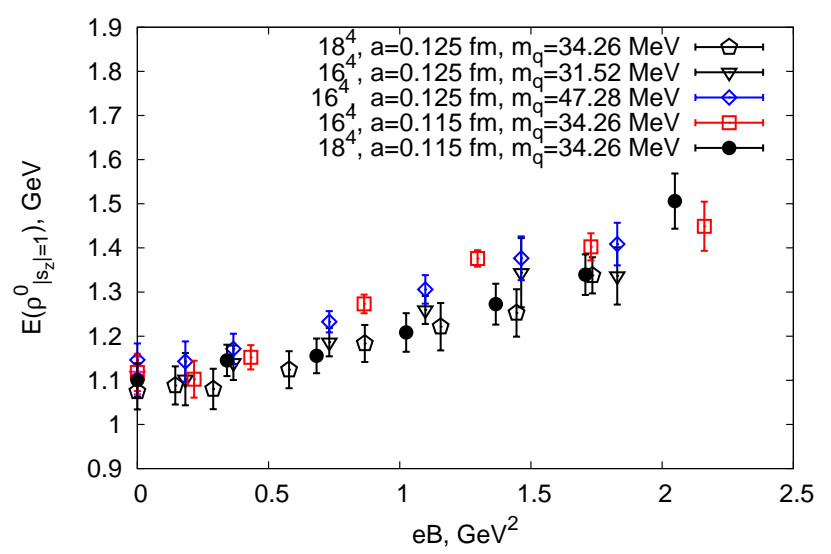

Figure 2: The same that in Fig.1 but for non-zero spin $s_{z}= \pm 1$ as a function of magnetic field for lattice volumes $16^{4}$ and $18^{4}$, lattice spacings $a=0.115$, fm and $0.125 \mathrm{fm}$ and various bare quark masses.

made in the $S U$ (2) lattice gauge theory in our previous work [16].

The energy levels of free charged pointlike particle in a background magnetic field parallel to 


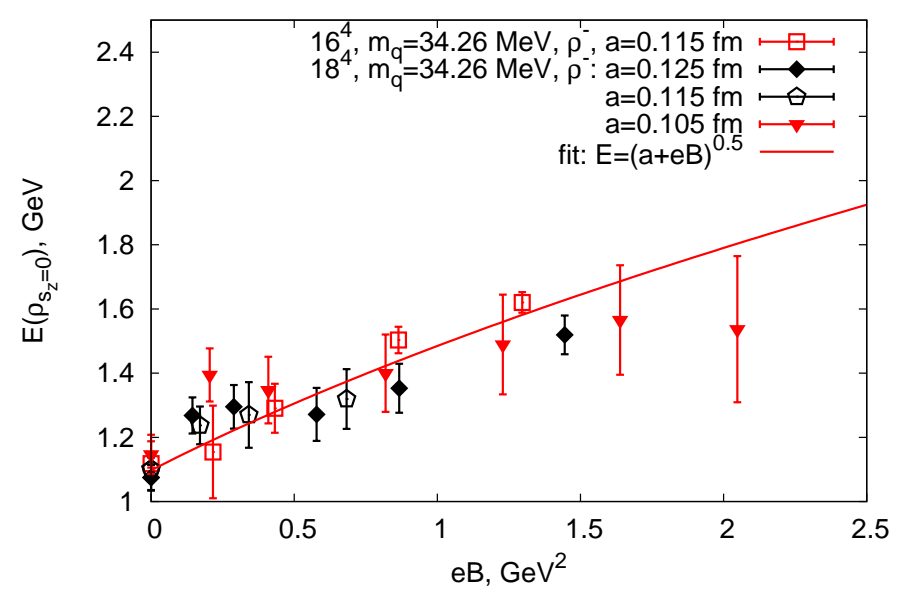

Figure 3: The ground state energy of the charged vector meson $\rho^{-}$with zero spin $s_{z}=0$ as a function of magnetic field for $16^{4}$ and $18^{4}$ lattices, $a=0.105$, fm, $a=0.115$, fm and $0.125 \mathrm{fm}$ and various bare quark masses.

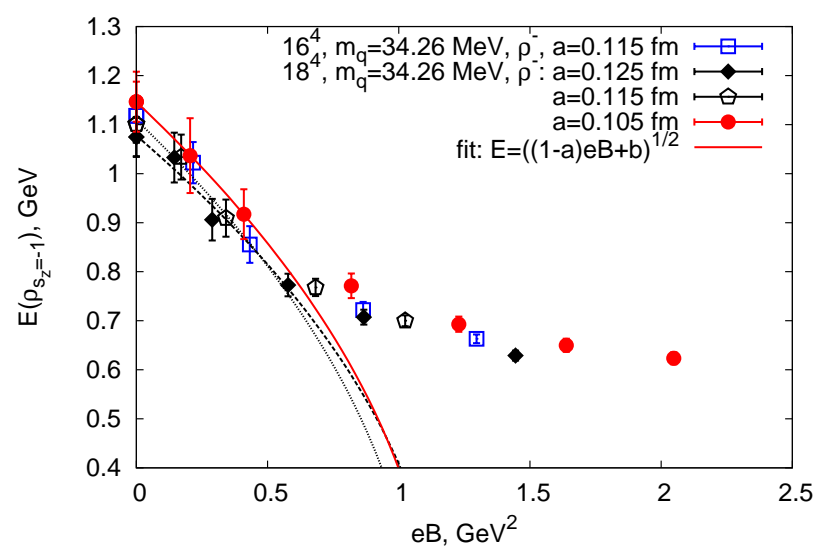

Figure 4: The same that in Fig.3 but for $\rho^{-}$meson with spin $s_{z}=-1$ versus the field value for lattice volumes $16^{4}$ and $18^{4}$, lattice spacings $a=0.105$, fm, $a=0.115$,fm and $0.125 \mathrm{fm}$ and various bare quark masses. Fits are made for the $18^{4}$ lattice volume; solid line corresponds to the $a=0.105 \mathrm{fm}$ lattice spacing, dot dashed line is for $0.115 \mathrm{fm}$, dashed line is for $a=0.125 \mathrm{fm}$.

$z$ axis

$$
E^{2}=p_{z}^{2}+(2 n+1)|q B|-g s_{z} q B+m^{2}(B=0)
$$

where $p$ is the momentum, $n$ is the number of energy level, $g$-factor characterizes magnetic properties of the particle, $q$ is the charge of the particle, $m^{2}(B=0)$ is the particle mass at $B=0$. In our case $p=0, n=0$ and $g$ and $m^{2}(B=0)$ are the parameters of the fitting function. This formula doesn't take into account polarizability of the particle, so has to work at not very large magnetic fields. 


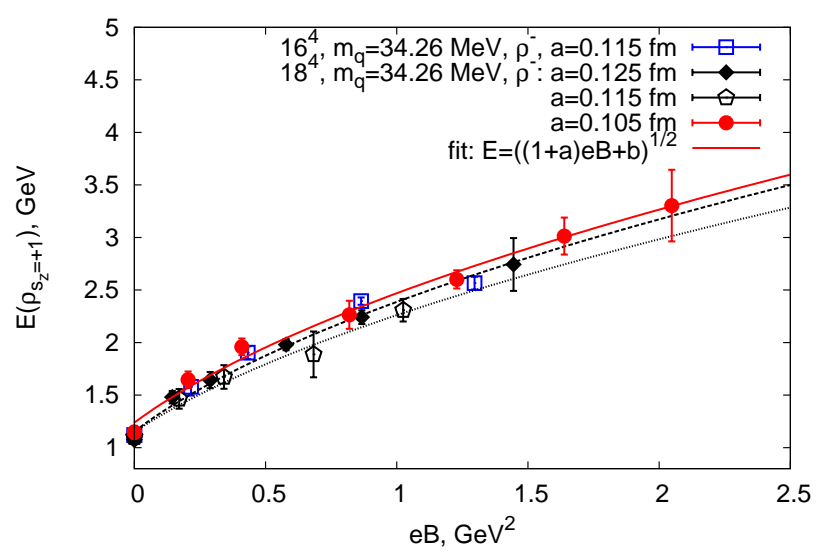

Figure 5: The same that in Fig.4 but for spin projection $s_{z}=+1$ versus the magnetic field value.

Fig. 3 depicts the mass of charged vector $\rho$ meson with $s_{z}=0$. We cannot distinguish negative and positive vector $\rho$ mesons on the lattice because the exchange of the particle charge to the opposite is equivalent to the exchange of $B$ to $-B$ which gives the same masses for $s_{z}=0$. This is in accordance with (3.5). We make a fit $E=\sqrt{a+e B}$ of the data for $18^{4}$ lattice volume and $a=0.105 \mathrm{fm}$ lattice spacing, $a=m^{2}(B=0)$ is a fit parameter. In spite of lack of statistics the data agree with the fit.

We shows the energies of charged $\rho^{-}$with spin projections $s_{z}=-1$ and $s_{z}=+1$ in Figs. 4 and 5 correspondingly. The energy of the $\rho^{-}$ground state with $s_{z}=-1$ decreases with the field value. The data agree with fit $E=\sqrt{(1-a) e B+b}$ for $e B \in\left[0,0.6 \mathrm{GeV}^{2}\right]$, at large magnetic field field the decrease became slower. We consider this effect as the result of non-zero polarizability of charged $\rho$ meson. The energy of $\rho^{-}$ground state with $s_{z}=+1$ increases with the field value. The function $E=\sqrt{(1+a) e B+b}$ gives the excellent fits for the all presented data.

During calculations we are limited to a small lattice spacing and therefore we can not explore too large values of the magnetic fields. As a result of simple estimates we obtain the value of the magnetic field $2.9 \mathrm{GeV}^{2}$ for lattice spacing $a=0.115 \mathrm{fm}$ and $2.5 \mathrm{Gev}^{2}$ for $a=0.125 \mathrm{fm}$, when the lattice spacing effects have to become appreciable.

\section{Conclusions}

We explore the behaviour of the masses of vector $\rho$ in $S U$ (3) lattice gauge theory. We found that masses of neutral vector $\rho^{0}$ mesons with zero and non-zero spin projection on the direction of the magnetic field differ from each other. Masses with $s_{z}=0$ decrease with increasing magnetic field, while the masses with $s_{z}= \pm 1$ increase under the same conditions. We consider this phenomenon as a result of the anisotropy produced by the strong magnetic field. We did not find condensation of neutral mesons, i.e. any indications of the existence of a phase of superfluidity in the confinement phase.

The masses of charged $\rho$ mesons cohere with the behaviour of Landau levels at not very large magnetic fields. We didn't observe some evidences in favour of condensation of charged vector 
mesons in the considered range of fields. Condensation of charged $\rho$ mesons might indicate the existence of superconductivity in QCD at high magnetic fields. The existence of a superconducting phase in QCD at high values of the magnetic field B [11] is still a hot topic for discussions.

\section{Acknowledgments}

This work was carried out with the financial support of Grant of President MK-6264.2014.2 and FRRC grant of Rosatom SAEC and Helmholtz Assotiation. The authors are grateful to FAIRITEP supercomputer center where these numerical calculations were performed.

\section{References}

[1] Grasso D and Rubinstein H R 2001 Phys. Rept. 348163 arXiv: astro-ph/0009061

[2] Skokov V, Illarionov A and Toneev V 2009 Int. J. Mod. Phys. A 245925 arXiv: 0907.1396[nucl-th]

[3] Bruckmann Falk, Endrodi Gergely and Kovacs Tamas 2013 JHEP 04112 arXiv: 1303.3972 [hep-lat]

[4] M. D’Elia, S. Mukherjee, F. Sanfilippo, Phys. Rev. D 82: 051501 (2010), arXiv: 1005.5365v2 [hep-lat].

[5] G.S. Bali, F. Bruckman, G. Endrodi, Z. Fodor, S.D. Katz, S. Krieg, A. Schafer, K.K. Szabo, JHEP, 02, 044 (2012), arXiv: 1111.4956 [hep-lat].

[6] Bornyakov V.G., Buividovich P.V.,Cundy N.,Kochetkov O.A. and SchÃd'fer A. 2014 Phys. Rev. D 90 034501 arXiv: 1312.5628 [hep-lat]

[7] C. Bonatti, M. D’Elia, M. Mariti, F. Negro, F. Sanfilippo, PoS Lattice 2013, arXiv:1312.5070.

[8] C. Bonatti, M. D’Elia, M. Mariti, F. Negro, F. Sanfilippo, arXiv: 1310.8656.

[9] G.S. Bali, F. Bruckman, G. Endrodi, A. Schaefer, PoS Lattice 2013, arXiv: 1310.8145.

[10] L. Levkova and C. DeTar, arXiv: 1309.1142 [hep-lat].

[11] Chernodub M N 2010 Phys. Rev. D 82 085011, arXiv: 1008.1055 [hep-ph]

[12] Chernodub M N 2011 Phys. Rev. Lett. 106142003 arXiv: 1101.0117v2 [hep-ph]

[13] M.A. Andreichikov, B.O. Kerbikov, V.D. Orlovsky, Yu.A. Simonov, arXiv: 1304.2533 [hep-ph].

[14] Y. Hidaka, A. Yamamoto, Phys. Rev. D 87, 094502 (2013), arXiv:1209.0007 [hep-ph].

[15] Liu H, Yu L and Huang M arXiv:1408.1318 [hep-ph]

[16] Luschevskaya E V, Larina O V 2014 it Nucl. Phys. B 884 1-16 arXiv: 1203.5699 [hep-lat]

[17] Bornyakov V G, Ilgenfritz E-M and Müller-Preussker M 2005 Phys. Rev. D 72054511 hep-lat/0507021 